\title{
Understanding the stretching mechanism of spiral- island configurations for highly stretchable elecronics
}

\author{
Nadeem Qaiser \\ MMH Labs \\ Electrical Engineering Department, King \\ Abdullah University of Science and \\ Technology (KAUST) Thuwal, Saudi Arabia \\ nadeem.qaiser@kaust.edu.sa
}

\author{
Sherjeel M. Khan \\ MMH Labs \\ Electrical Engineering Department, King \\ Abdullah University of Science and \\ Technology (KAUST) Thuwal, Saudi Arabia \\ sherjeel.khan@kaust.edu.sa
}

\author{
Muhammad M. Hussain \\ MMH Labs \\ Electrical Engineering Department, King \\ Abdullah University of Science and \\ Technology (KAUST) Thuwal, Saudi Arabia \\ muhammadmustafa.hussain@kaust.edu.sa
}

\begin{abstract}
Spiral-island is commonly used configuration for highly stretchable and flexible electronics. Here we show the elongations and stress behaviors of spiral-island system. We use the numerical modeling to reveal the stress states along the arms of spirals, especially when the spirals connect in series and as well as triangular configurations between islands. We stretched out the spirals up to $1000 \mu \mathrm{m}$ and compared the behaviors for single, two and four spirals connected in series. Numerical calculations show that during stretching, the stress is higher near the region of arm's start i.e. end that is connected to inner circle.. Our result also show that final elongation of spiral and arm's stresses depend on the angular position and the no. of spirals connected in series. We use the 3D printer to manufacture the spiral-island for triangular configuration and compare the elongation of spirals, which confirm our numerical results.
\end{abstract}

Keywords - mechanical deformations, numerical analysis, spiral interconnect, tensile testing, stretchable electronics

\section{INTRODUCTION}

Currently, flexible and stretchable electronics play a pivoting role in different areas, which include optoelectronics, bio-inspired health care devices, soft robotics used for surgical operations, and wearable communication devices.[1]-[3] In addition to flexibility, stretchable electronics have high performance and low cost as compared to rigid counterparts. Previously, M. M. Hussain and his team has successfully developed the CMOS compatible integration strategy for flexible devices by using the thinner Si.[4], [5] Stretchable and flexible electronics induce large deformations during their operation and thus need a design, which can accommodate the deformations. For this purpose, a spiral-island configuration is commonly used, wherein the electronic components are placed onto the island, where the spiral connected between islands (as shown in figure 1) accommodates all of the deformations during the stretching. Q. Nadeem et al. reported the mechanical response of these spiral interconnects for different geometrical morphologies.[6] However, for better understanding of these spiral interconnect designs, the underlying mechanics in terms of stress history along the arm of spiral is still needs to be studied. Here we show the mechanical response of spiral connected between islands, when stretched out by prescribing the displacement of $1000 \mu \mathrm{m}$. We use the numerical tool i.e. Finite Element Method (FEM) to calculate the elongations and stresses on the surface of arms. We also have revealed the effect of adding the spirals in series between the islands. The angular position of spiral alters the deformation behaviors of that respective spiral. We have fairly corroborated our FEM based elongation results with tensile testing results of $3 \mathrm{D}$ printed samples.

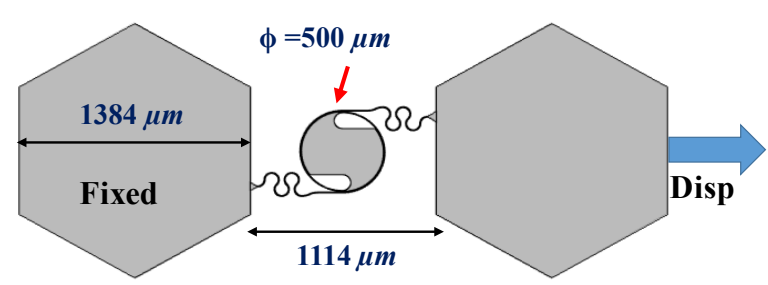

Figure 1. Single-turn spiral connected between two islands

\section{FEM ANALYSIS AND TESTING OF 3D PRINTED SAMPLES}

The structure and sizes of the island-spiral configuration is show in figure 1. With one end fixed (left island), when the right island is stretched up to $1000 \mu \mathrm{m}$, the spiral will start to unwind and accommodates the large deformations. In order to demonstrate the effect of spiral position, we used multiple spiral-islands by connecting them in series and at angular position. We used the SOLIDWOKS ${ }^{\mathrm{TM}}$ to design the one, two, and four spirals in series and triangular configuration. In order to calculate the stresses in the structure, we implemented the commercially available tool ABAQUS ${ }^{\mathrm{TM}}$. We imported the 3D solid designs in ABAQUS ${ }^{\mathrm{TM}}$ and prescribed the displacement. Once the spiral starts to open, the arm of spiral experiences the stresses. Naturally, these stresses are not constant along the arm. We extracted the average von Misses stress along the half of the arm, since the behavior of other half arm would not differ. We have defined the stretchability (stretch) as the final separation or gap between islands/initial gaps. To converge the solution, we used the fine mesh near stress concentration regions. Material properties of single crystalline, isotropic $\mathrm{Si}$ were used.

We compared the FEM results of triangular configuration with the $3 \mathrm{D}$ printed samples. We used the 3D printer (Xeed from Leapfrog Inc.) to manufacture the triangular configuration. We used a biodegradable plastic filament Poly Lactic Acid (PLA) with $1.75 \mathrm{~mm}$ size. To get the required tensile stretching, we designed a rack and pinion gear assembly 
and used servo motor, which converted the circular motion into linear and provided the tensile testing capability.

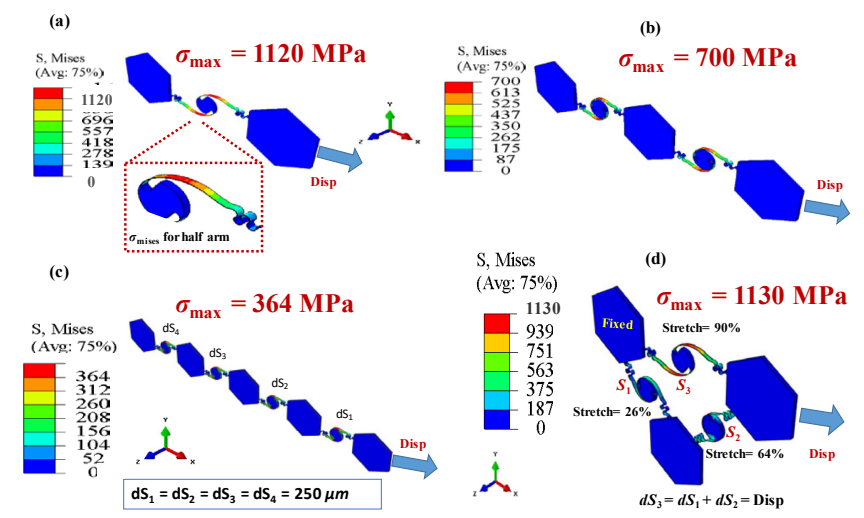

Figure 2. 3D stress contours under prescribed displacement of $1000 \mu \mathrm{m}$ in various spiral interconnect configurations. (a) single-spiral case, (b, c) two and four-spirals connected in series between islands, respectively, and (d) triangular configurations

\section{RESULTS AND DISCUSSIONS}

\section{A. Spirals connected in series}

Figure 2 shows the 3D stress contours under the prescribed displacement of $1000 \mu \mathrm{m}$. Figure 2a represents the configuration of single spiral connected between islands. For single spiral case, maximum von Misses stress $\left(\sigma_{\max }\right)$ reaches to $1120 \mathrm{MPa}$. Figure $2 \mathrm{~b}$ and $2 \mathrm{c}$ shows the $3 \mathrm{D}$ stress contours for two and four spiral in series and shows that all of the spirals share the prescribed displacement equally. The average von Misses stress (actual and polynomial fitted curves) along the half of the arm ( $\left.\sigma_{\text {misses }}\right)$ are shown in figure 3. It is clear from the figure that stress decreases when another spiral is added in series. For instance, for two and four spiral case, the stress decreases from $1120 \mathrm{MPa}$ to $700 \mathrm{MPa}$ and then further to 364 $\mathrm{MPa}$, respectively.

\section{B. Triangular configuration: FEM and experimental}

Figure $2 \mathrm{~d}$ shows the results for triangular configuration, where the spirals are connected at angles. It is worthy to mention that the spiral which is connected directly between displaced and fixed island, shows the maximum stretchability and thus the stresses are also higher in that particular spiral. Likewise, the stresses for each of spiral in triangular configuration is shown in figure $3 \mathrm{~b}$, which show that the stresses depend on the angular position of the spiral. However, the displacement is shared among all of spiral in such a manner that $\mathrm{dS}_{3}=\mathrm{dS}_{1}+\mathrm{dS}_{2}=$ prescribed displacement. Figure 4a shows the $3 \mathrm{D}$ printed samples (macro scale i.e. up to $\sim \mathrm{cm}$ scale) before and after the tensile testing. For fair comparison between micro and macro sample, we used the normalized separation or gap. It is clearly seen that the elongation calculated by FEM fairly corroborate the experimental results of tensile testing.

\section{CONCLUSION}

We have shown the stretching mechanism of spirals, by FEM and experiments, connected in series and angular positions, which is handy for highly stretchable electronics. FEM results show that spirals connected in series, share the displacement equally. The angular position of spiral alters the deformation behaviors of that respective spiral. Stretchability and von Misses stresses decreases when additional spiral are connected in series and depends on angular position when connected at angle.
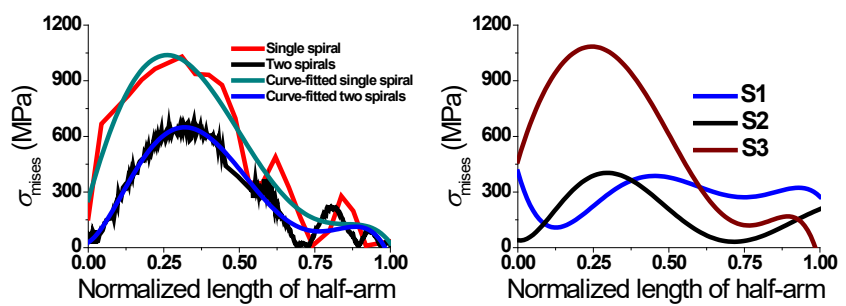

Figure 3 (a) actual and curve fitted von Misses stress along the normalized length of half-arm of spiral for single and two spiral case, and (b) for triangular configuration. Under same displacement, stress decreases when additional spiral is added in the system.

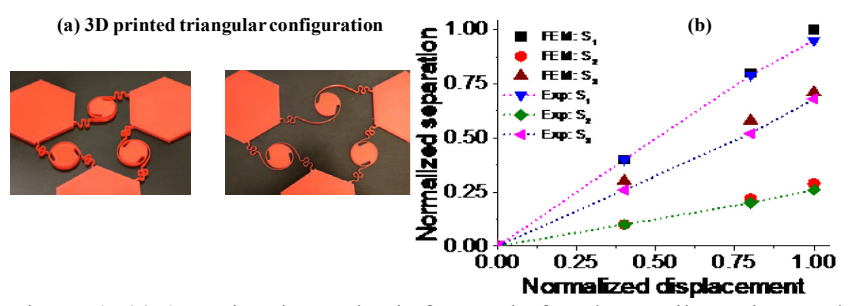

Figure 4. (a) 3D printed samples before and after the tensile testing, and (b) comparison of experimental and numerical results

\section{REFERENCES}

[1] S. Choi, H. Lee, R. Ghaffari, T. Hyeon, and D.-H. Kim, "Recent Advances in Flexible and Stretchable Bio-Electronic Devices Integrated with Nanomaterials," Adv. Mater., vol. 28, no. 22, pp. 4203-4218, Jun. 2016.

[2] S.-I. Park et al., "Printed Assemblies of Inorganic Light-Emitting Diodes for Deformable and Semitransparent Displays," Science (80.)., vol. 325, no. 5943, 2009.

[3] J. Viventi et al., "A Conformal, Bio-Interfaced Class of Silicon Electronics for Mapping Cardiac Electrophysiology,” Sci. Transl. Med., vol. 2, no. 24, 2010

[4] J. P. Rojas, A. Arevalo, I. G. Foulds, and M. M. Hussain, "Design and characterization of ultra-stretchable monolithic silicon fabric," Appl. Phys. Lett., vol. 105, no. 15, p. 154101, Oct. 2014.

[5] J. P. Rojas et al., "Transformational Silicon Electronics," ACS Nano, vol. 8, no. 2, pp. 1468-1474, Feb. 2014.

[6] N. Qaiser, S. M. Khan, M. Nour, M. U. Rehman, J. P. Rojas, and M. M. Hussain, "Mechanical response of spiral interconnect arrays for highly stretchable electronics," Appl. Phys. Lett., vol. 111, no. 21, p. 214102, Nov. 2017. 